\title{
Predicting the Dynamics of Network Connectivity in the Neocortex
}

\author{
Yonatan Loewenstein, ${ }^{1}$-Uri Yanover, ${ }^{2}$ and Simon Rumpel ${ }^{3,4}$ \\ ${ }^{1}$ Edmond and Lily Safra Center for Brain Sciences, Department of Neurobiology, Alexander Silberman Institute of Life Sciences, Department of Cognitive \\ Science and the Ferdermann Center for the Study of Rationality, and ${ }^{2}$ Rachel and Selim Benin School of Computer Science and Engineering, Hebrew \\ University of Jerusalem, 91905 Jerusalem, Israel, ${ }^{3}$ Research Institute of Molecular Pathology, 1030 Vienna, Austria, and ${ }^{4}$ Institute of Physiology, Focus \\ Program Translational Neurosciences, University Medical Center, Johannes Gutenberg University Mainz, 55099 Mainz, Germany
}

Dynamic remodeling of connectivity is a fundamental feature of neocortical circuits. Unraveling the principles underlying these dynamics is essential for the understanding of how neuronal circuits give rise to computations. Moreover, as complete descriptions of the wiring diagram in cortical tissues are becoming available, deciphering the dynamic elements in these diagrams is crucial for relating them to cortical function. Here, we used chronic in vivo two-photon imaging to longitudinally follow a few thousand dendritic spines in the mouse auditory cortex to study the determinants of these spines' lifetimes. We applied nonlinear regression to quantify the independent contribution of spine age and several morphological parameters to the prediction of the future survival of a spine. We show that spine age, size, and geometry are parameters that can provide independent contributions to the prediction of the longevity of a synaptic connection. In addition, we use this framework to emulate a serial sectioning electron microscopy experiment and demonstrate how incorporation of morphological information of dendritic spines from a single time-point allows estimation of future connectivity states. The distinction between predictable and nonpredictable connectivity changes may be used in the future to identify the specific adaptations of neuronal circuits to environmental changes. The full dataset is publicly available for further analysis.

Key words: connectome; dendritic spines; synaptic dynamics

\section{Significance Statement}

The neural architecture in the neocortex exhibits constant remodeling. The functional consequences of these modifications are poorly understood, in particular because the determinants of these changes are largely unknown. Here, we aimed to identify those modifications that are predictable from current network state. To that goal, we repeatedly imaged thousands of dendritic spines in the auditory cortex of mice to assess the morphology and lifetimes of synaptic connections. We developed models based on morphological features of dendritic spines that allow predicting future turnover of synaptic connections. The dynamic models presented in this paper provide a quantitative framework for adding putative temporal dynamics to the static description of a neuronal circuit from single time-point connectomics experiments.

\section{Introduction}

The structure of neuronal circuits is a major determinant of their computational function. In recent years, considerable efforts

\footnotetext{
Received July 15, 2014; revised July 8, 2015; accepted July 14, 2015.

Author contributions:Y.L., U.Y., and S.R. designed research;Y.L.,U.Y., and S.R. performed research;Y.L., U.Y., and S.R. analyzed data; Y.L., U.Y., and S.R. wrote the paper.

This work was supported by the Ministry of Science and Technology, Israel, the Ministry of Foreign and European Affairs, the Ministry of Higher Education and Research, France, and the Gatsby Charitable Foundation to Y.L. and U.Y., and the Boehringer Ingelheim GmbH to S.R. We thank Anthony Zador and Roberto Malinow for support and encouragement during the early phase of the project; and Gianluigi Mongillo for comments on the manuscript.

The authors declare no competing financial interests.

Correspondence should be addressed to either of the following: Dr. Simon Rumpel, Institute of Physiology, Focus Program Translational Neurosciences, University Medical Center, Johannes Gutenberg University Mainz, 55099 Mainz, Germany, E-mail: sirumpel@uni-mainz.de; or Dr. Yonatan Loewenstein, Edmond and Lily Safra Center for Brain Sciences, Department of Neurobiology, Alexander Silberman Institute of Life Sciences, Department of Cogni-
}

have been made to extract information about neuronal connectivity in histological samples. In particular, serial-sectioning electron microscopy (ssEM) reconstruction has been successful in determining the structure of neuronal networks with single synapse resolution (Lichtman and Denk, 2011). Although this approach was used so far for the analysis of different neuronal circuits, including the neuronal circuit in Caenorhabditis elegans (White et al., 1986), circuits in fly visual system (Takemura et al., 2013), in the mammalian retina (Briggman et al., 2011; Helmstaedter et al., 2013), and fractions of the neocortex (Bock et al.,

tive Science and the Ferdermann Center for the Study of Rationality, Hebrew University of Jerusalem, 91905 Jerusalem, Israel, E-mail: yonatan@huji.ac.il.

DOI:10.1523/JNEUROSCI.2917-14.2015

Copyright $\odot 2015$ the authors $\quad 0270-6474 / 15 / 3512535-10 \$ 15.00 / 0$ 
2011), it is likely that, with the advancement of technology, increasingly larger brain regions will become amenable for reconstruction in the foreseeable future. The knowledge of the socalled "connectivity matrix," which specifies the existence and strength of connections between all neurons in a brain region, holds the promise for novel insights in our understanding of the activity and function of neuronal circuits.

However, inferring the function of the neuronal circuit from its connectivity matrix remains a challenge (Seung, 2009; Denk et al., 2012; Bargmann and Marder, 2013). One factor that fundamentally complicates the interpretation of connectomic data from a single time-point is the fact that, in the living brain, connectivity matrices are dynamic. This is particularly evident in chronic imaging of dendritic spines, on which most of neocortical excitatory synapses reside (Beaulieu and Colonnier, 1985; Knott et al., 2006; Arellano et al., 2007b). First, spine sizes change at multiple time-scales, suggesting that the efficacies of the corresponding synapses undergo constant changes (Minerbi et al., 2009; Kasai et al., 2010; Loewenstein et al., 2011). Second, spines exhibit substantial turnover, which indicates that the formation and elimination of synapses is an ongoing process (Holtmaat and Svoboda, 2009). Both these processes are observed not only during development but also in the adult brain.

Dissociating the more stable elements from the less stable ones in the connectivity matrix can be useful when relating the neuronal architecture to its function (Moczulska et al., 2013). For example, it is thought that the ability to store memories for long periods of time depends on the stability of specific patterns of synaptic connections (Hübener and Bonhoeffer, 2010; Caroni et al., 2012). Therefore, it is likely that stability of some elements of the matrix enable long-term memories (Xu et al., 2009; Yang et al., 2009; Moczulska et al., 2013). On the other hand, it is difficult to see how the volatile elements of the connectivity matrix participate in the long-term storage of memories.

Previous spine imaging studies have demonstrated that spines differ in their dynamics. For example, it has been shown that the age (Grutzendler et al., 2002; Trachtenberg et al., 2002; Holtmaat et al., 2005; Zuo et al., 2005; Keck et al., 2008) and size (Grutzendler et al., 2002; Holtmaat et al., 2005; Zuo et al., 2005; Majewska et al., 2006) of the spine are negatively correlated with its turnover rate. This suggests that distinct morphological features of a spine could be used as predictors of their stability. The goal of this study is to identify and quantify the power of various morphological features to predict future network reconfigurations.

\section{Materials and Methods}

We used six male adult ( $\sim 6$ months old) in-house bred mice of the GFP-M transgenic line (Tg(Thy1-EGFP)MJrs/J) selected for sparse GFP expression in the cortex (Feng et al., 2000). All experiments were performed at Cold Spring Harbor Laboratory, in strict compliance with the animal use and care guidelines of Cold Spring Harbor Laboratory. The procedures for implantation of a glass window in the cranium and in vivo two-photon imaging for the acquisition of this dataset have been described in detail previously (Loewenstein et al., 2011).

Image analysis. For image analysis, "best projections" of all dendrites for all time-points were constructed that allowed identification of spines at a given time-point and indexing of identical spines along time (Holtmaat et al., 2005). A custom-written MATLAB (The MathWorks) software was used for extraction of morphological features of identified spines. Comparison of images of dendritic spines acquired by twophoton microscopy and subsequent ssEM reconstruction highlights the fact that the estimation of morphological parameters in two-photon data is less precise due to the poorer resolution, in particular for the axial dimension (Knott et al., 2006; Cane et al., 2014). To minimize this effect, we focused our analysis on those spines, which extended laterally from the dendrite approximately parallel to the imaging plane. For $>72 \%$ of analyzed spines, the estimated center of the adjacent dendrite was within $0.5 \mu \mathrm{m}$ along the $z$-axis. For the measurement of $V, S$, and $D$, the $2 \mathrm{D}$ image plane, in which the spine brightness was highest was considered. The morphological parameter $V$ was defined as the common logarithm of the integrated spine intensity, as described in detail previously (Loewenstein et al., 2011). It has previously been shown that integrated spine intensity in in vivo two-photon imaging is tightly correlated with the volume of a spine as subsequently estimated by ssEM reconstruction (Grutzendler et al., 2002; Trachtenberg et al., 2002; Holtmaat et al., 2005; Zuo et al., 2005; Keck et al., 2008). Furthermore, there is good evidence that the spine volume can serve as proxy for the functional strength of a synaptic connection (Bhatt et al., 2009; Holtmaat and Svoboda, 2009; Holtmaat et al., 2009; Xu et al., 2009). For the purpose of this study, we extracted in addition two parameters that describe the shape $S$ of a spine and the distance $D$ of its center of mass to the dendrite. To obtain $S$, we performed a principal component analysis of the $2 \mathrm{D}$ pixel map associated to the spine, where each pixel was weighted by its brightness. Denoting the larger and smaller eigenvalues by $\lambda_{1}$ and $\lambda_{2}$, respectively, $S=\frac{\lambda_{1}-\lambda_{2}}{\lambda_{1}+\lambda_{2}}$. The parameter $D$ was calculated as the shortest distance between the center of mass of the spine-associated pixels and the ridge along the dendrite as defined by a watershed algorithm in arbitrary units. Thus, $D$ is a measure of the length of a spine. An advantage of this method is that it does not rely on the determination of spine boundaries. However, it may be affected by the geometry of the spine and the spine's relative orientation to the dendrite.

Statistical analysis. To compute the standard error of the mean (SEM) for the fraction of newly formed spines in our dataset, $p_{\text {new }}$, we considered every spine imaged as an independent Bernoulli process. Thus, the most likely mean is the empirical frequency $p_{n e w}=$ $2,268 / 7,279=0.312$, and the SEM is given by SEM = $\sqrt{p_{\text {new }} \cdot\left(1-p_{\text {new }}\right) /(7,279-1)=} 0.005$. To estimate the confidence interval (CI) in the values of $\gamma$, we solved Equation 1 in Results numerically for $p_{\text {new }}=0.312+0.005$ and for $p_{\text {new }}=0.312-0.005$ and used the resultant values of $\gamma$ as the CIs for $\gamma$.

The parameters used in Figures 3, 5, and 6, and Table 1 are those that minimize the square difference (averaged over the observations) between the predicted probabilities of survival ( $p$ in Eq. 2 ) and the empirical observations of spines dynamics ( 1 , survived; 0 , did not survive). Replacing the least square error objective function with a maximum likelihood score did not substantially change the estimated parameters.

To estimate the confidence in the estimated parameters (Figures 3, 5; Table 1), we used nonparametric bootstrapping: we resampled the spines, with replacement, 10,000 times and for each of these realizations we repeated the regression. The CIs of these parameters are denoted by error bars in Figures 3 and 5 and by the error margins in Table 1. Comparable results were obtained when parametric bootstrapping was applied.

To compute the log-likelihoods of Figure 6, we used the models to compute the survival probabilities of individual spines and used these probabilities to compute the log-likelihoods of the data: $L=\sum_{i}\left(a_{i} \cdot \log _{2}\left(p_{i}\right)+\left(1-a_{i}\right) \cdot \log _{2}\left(1-p_{i}\right)\right)$ where the sum is over all spines, $p_{i}$ is the probability of survival of spine $i$ and $a_{i}$ is in index variable that is equal to 1 if the spine survived and is equal to 0 otherwise. To avoid overfitting, we used the method of "leave one out": the survival probability for each of the spines was estimated using a model that was trained on all other spines (not including the spine whose lifetime was predicted). Comparable log-likelihoods were obtained using the twofold cross-validation technique, in which the data were divided into two sets of equal size: one set was used for training the models and the other for estimating their log-likelihoods.

CIs for likelihood were estimated using nonparametric bootstrapping: we resampled the spines, with replacement, 10,000 times and for each of these realizations we repeated the log-likelihood analysis (using the "leave one out" method described above). The error bars in Figure $6 B$ correspond to the standard deviations (SDs) of the resultant log likeli- 

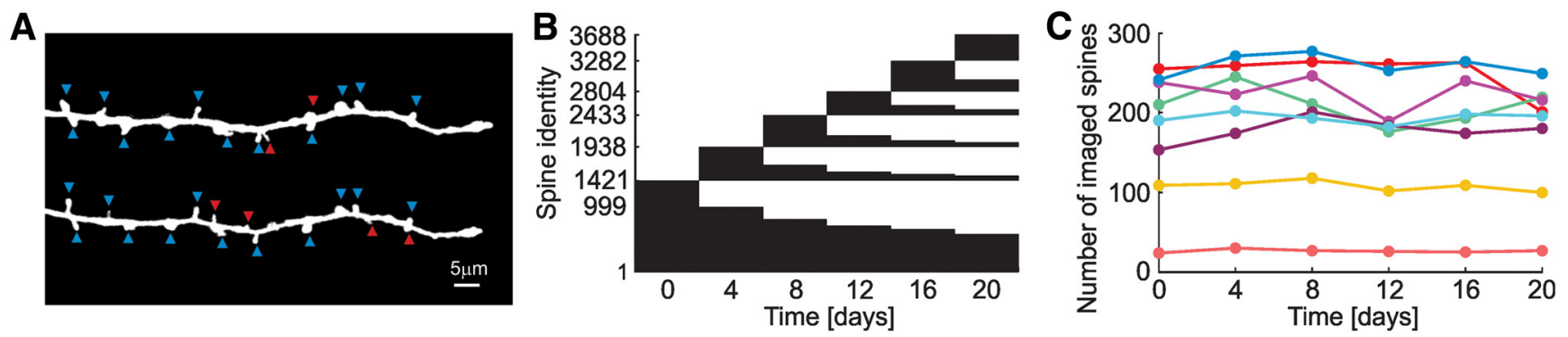

Figure 1. In vivo imaging of dendrites and spines. $\boldsymbol{A}$, Two "best projection" images (Holtmaat et al., 2005) of the tip of the same dendrite imaged at an interval of $4 \mathrm{~d}$. Blue arrowheads indicate spines present at both time-points. Red arrowheads indicate spines found only on one of the days. $\boldsymbol{B}$, Lifetime plot of all 3688 imaged spines. Presence of a spine is indicated by a thin horizontal black line on a given day. For example, spines 1-1420 are those observed in the first imaging session; spines 1-998 are those observed in at least the first and the second imaging session; spines 1421-1937 are those observed for the first time in the second imaging session; and so on. C, Total number of spines imaged on a given day per neuron (indicated by different colors).

hoods. Comparable results were obtained when parametric bootstrapping was applied.

To compare the different models, we used the fact that, for nested models, the difference in the (natural) log-likelihood of the models, multiplied by 2 , is $\chi^{2}$ distributed, with degrees of freedom equal to the difference in the number of parameters (Wilks, 1938; Dickhaus and Royen, 2015). The (natural) likelihoods of the different models are depicted in Table 1 . We used the same approach to validate that including the distance variable beyond the shape variable is justified.

\section{Results}

Imaging dendritic spines in the mouse auditory cortex in vivo We imaged and analyzed 3688 spines from eight neurons of the auditory cortex in six mice expressing GFP in a subset of pyramidal neurons (Feng et al., 2000) using chronic two-photon imaging through a cranial window (Fig. $1 A$ ). The full details of the experimental procedures have been described previously (Loewenstein et al., 2011), where we analyzed part of this dataset to address a different question. We observed substantial spine turnover, indicating that the architecture of the neuronal circuits in the auditory cortex is dynamic (Fig. 1B). Indeed, 31\% $\pm 1 \%$ (SEM) of the spines in a given imaging session were not detected in the previous imaging session; and, similarly, $31 \pm 1 \%$ (SEM) of the spines identified in an imaging session were no longer found in the next imaging session. The total number of spines imaged on a given day was almost constant (Fig. $1 C$ ). It should be noted that previous studies have reported a wide range of turnover rates, and the rates reported in this study are at the higher end. Differences between rates have been attributed to the cortical region, age of the animal, and differences in the imaging technique (Bhatt et al., 2009; Holtmaat and Svoboda, 2009; Holtmaat et al., 2009; Xu et al., 2009). One major factor contributing to our relatively high estimate of turnover rates is that we did not attempt to distinguish between filopodia and spines.

The goal of this paper is to characterize the independent contributions of different spine features to their stability. The results are presented in the following order: First, we characterize the dependence of spine turnover on the age of the spine. Second, we quantify how the size and the age of the spine independently contribute to the prediction of its lifetime. Third, we construct a comprehensive model that predicts the lifetime of a spine based on its age, size, and additional morphological features. Fourth, we use this framework to estimate the power of a single time-point ssEM experiment to identify stable and volatile components of the network architecture, when age information is omitted and only the morphological features are incorporated.
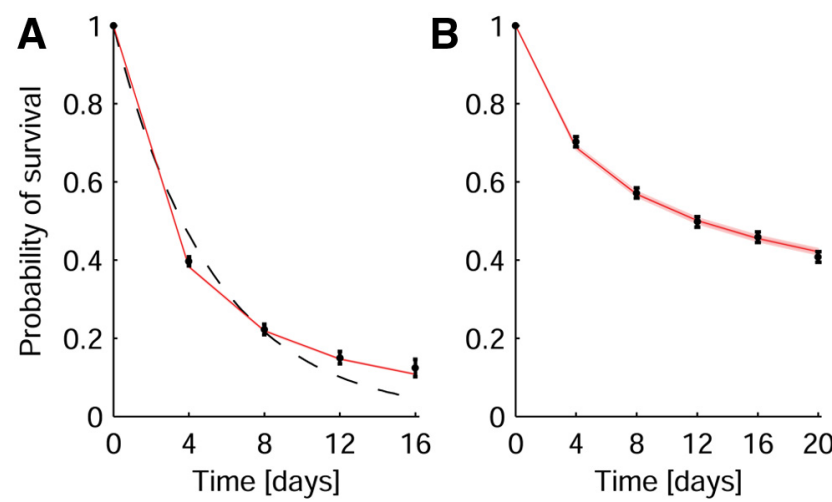

Figure 2. Lifetime dynamics of spines follow a power law. $A$, Survival plot of newly formed spines (corresponding to spines 1421-3281 in Fig. 1B). Circles represent the ratio of spines present one, two, three, and four imaging sessions out of the corresponding newly formed spines ( 739 of 1861, 308 of 1383, 153 of 1012, and 64 of 517, respectively). Dotted line indicates best exponential fit (time constant $5.2 \mathrm{~d}$ ). Red line and pink shade represents predicted survival plot and $\mathrm{Cl}, f(t)=(t+1)^{-\gamma}$, where $\gamma=1.384 \pm 0.008$ and $t$ is the imaging session. $\boldsymbol{B}$, Circles represent survival plot of spines observed in the first imaging session (corresponding to spines 1-1420 in Fig. 1B). Of the 1420 spines observed in the first imaging day, 998 , $812,707,651$, and 579 were still detected in the second, third, fourth, fifth, and sixth imaging sessions, respectively. Red line and pink shade (almost too thin to observe) represent predicted survival plot and Cl, respectively. Error bars (barely larger than the circle) indicate SEM.

\section{Power law description of spine turnover}

Before relating the morphological features of a spine to its lifetime, we quantified how the stability of a spine depends on its age (Grutzendler et al., 2002; Trachtenberg et al., 2002; Holtmaat et al., 2005; Zuo et al., 2005; Keck et al., 2008). We first considered newly formed spines, that is, spines that had not been observed on the first imaging session and appeared at some later timepoint during the experiment (Fig. $1 B$, spines 1421-3281). We plotted their survival probability curve (i.e., the fraction of newly formed spines surviving at least to the $t^{\text {th }}$ subsequent imaging session) (Fig. 2A). We found that $>60 \%$ of newly formed spines no longer existed after one imaging session. However, their disappearance rate was not constant. The longer the spine survived, the larger was the probability that it would survive to the next imaging session. This stabilization of spines with time is clearly evident when considering the significant deviation of the best exponential fit from the data points.

The survival plot of Figure $2 A$, in which the decay rate decreases with time is reminiscent of a power law decay, in which the probability that a spine survives to a given imaging session $t(t=0,1,2,3,4), f(t)$, is given by $f(t)=(t+1)^{-\gamma}$ and $\gamma>0$ is the decay parameter. However, it is difficult to assess a power law 
decay using a limited temporal range. Therefore, we used an indirect method that is based on the assumption that the dynamics of spine formation and removal is stationary. This assumption is based on our observation that the number of newly formed spines in an imaging session, the total number of spines and their ratio ( 517 of 1515,495 of 1537,371 of 1373,478 of 1466 , and 407 of 1388 for sessions 2-6, respectively) did not significantly change over the course of the experiment $(p>0.27, p>0.18$, and $p>0.37$, respectively, Student's $t$ test; see also Fig. $1 C$ ). The total number of spines $N$ in an imaging session is composed of all spines that survived to this imaging session. Therefore, assuming that the dynamics of spine formation and elimination is stationary, these $N$ spines can be grouped according to their age, such that $N=\sum_{t=0}^{\infty} n_{\text {new }} \cdot f(t)$, where $n_{\text {new }}$ is the number of observed spines that were not present in the previous imaging session. The fraction of newly formed spines is given by $p_{n e w}=\frac{n_{\text {new }}}{N}$ and, therefore, $p_{\text {new }}=\left(\sum_{t=0}^{\infty} f(t)\right)^{-1}$. Considering a power law survival probability function $f(t)=(t+1)^{-\gamma}$, the parameter $\gamma$ is uniquely determined by $p_{\text {new }}$ such that,

$$
p_{\text {new }}=\left(\sum_{t=0}^{\infty}(t+1) f^{-\gamma}\right)^{-1}
$$

Measuring the fraction of newly formed spines in our dataset, $p_{\text {new }}=0.312 \pm 0.005$ (2268 of 7279) and solving Equation 1 numerically, we found that $\gamma=1.384 \pm 0.008$. Plotting the predicted survival probability function $f(t)=(t+1)^{-\gamma}$ with $\gamma=1.384 \pm 0.008$ in Figure $2 A$, we found that a power law decay is a good approximation of the empirical data (all four data points were not statistically significantly different from the power law function, $p>0.22$, two-tailed binomial test). It is important to note that the predicted decay is not a fit of the empirically measured decay. Rather, it is a prediction that is based on the fraction of newly formed spines, $p_{\text {new }}$ and the assumptions of a power law decay and stationarity.

To further test the validity of Equation 1, we considered the lifetimes of spines that were observed in the first imaging session (spines 1-1420 in Fig. 1B). These spines were generated before the first observation; therefore, we have no direct information about their age composition. Their empirical lifetime plot is depicted in Figure $2 B$. The decay of these spines is significantly slower than that of the newly formed spines, likely due to the fact that many of them are older than newly formed spines. We assumed that the age composition of the population of spines observed in the first imaging session results from the same power law decay (Eq. $1 ; \gamma=1.384$ ) that describes the dynamics of newly formed spines (Fig. 2A). According to this, 0.31 of the spines were newly formed: $0.31 \cdot 2^{-1.38}=0.12$ were between 4 and 8 days old; $0.31 \cdot 3^{-1.38}=0.07$ were between 8 and 12 days old; and so on. After estimating the age composition of the spine population on a given day, we used Equation 1 to compute the predicted survival plot of the population present on the first imaging session. The theoretically predicted survival plot approximates the data well (Fig. $2 B ; p>0.27$, two-tailed binomial test for each of the five data points), indicating that the age-dependent turnover of the newly formed spines is comparable with the age-dependent turnover of the spines present in the first imaging session. Interestingly, a previous study has suggested, based on theoretical considerations, that the distribution of life times of newly created synapses matches a power law (Zheng et al., 2013). However, the value of the decay parameter in that study was substantially smaller $(\sim 0.5)$.

\section{Contribution of spine size and age to its stability}

To further characterize the determinants of spine stability, we considered the sizes of the spines. Previous studies have shown that smaller spines are more likely to disappear before the next imaging session than are larger spines (Grutzendler et al., 2002; Trachtenberg et al., 2002; Holtmaat et al., 2005; Zuo et al., 2005; Keck et al., 2008). Similarly, we find that the probability that a spine would survive to the next imaging session is a monotonously increasing function of its size (see below). However, because spine size and spine age are also correlated, at least during the initial time period after formation (Holtmaat et al., 2006) (see also below), we examined the independent contribution of the two factors to the prediction of the lifetime of the spines. To that goal, we grouped all spines according to their age. For each age group, we binned the spines according to their size into four classes of an equal number of spines. For each class, we calculated the survival probability of the spines by computing the fraction of spines that persisted to the next imaging session (Fig. $3 A$ ). The analysis reveals that conditioned on the age of the spine, the survival probability of larger spines is typically higher than that of smaller spines as indicated by the positive slope of the lines corresponding to the different age groups in Figure $3 A$. Moreover, for a given size of a spine, the average survival probability of older spines is higher than that of younger spines. This is reflected by the fact that the lines associated with the older spine groups are above the lines associated with the younger spine groups. These results clearly show that size and age independently contribute to the survival probabilities of spines.

To quantify the independent contribution of age and size to the survival probability of a spine, we used logistic regression to relate spines' turnover to their size and age. According to this model, the probability $p$ that a spine would survive to the next imaging session is a logistic function of $X$ as follows:

$$
p=\frac{e^{X}}{1+e^{X}}
$$

where $X$ is a linear combination of the age of the spine and its size in a logarithmic scale as follows:

$$
X=b_{i}+w_{v} \cdot \tilde{V}
$$

The parameter $b_{i}$ denotes the contribution of age of the spine to its survival such that, for all spines of equal age $i$, the age contribution is equal. The larger the value of $b_{i}$, the more stable the spine is. The parameter $w_{v}$ denotes the contribution of the size of the spine to its survival. The variable $\tilde{V}$ is the normalized size of the spine such that $\tilde{V}=\frac{V-\mu_{v}}{\sigma_{V}}$, where $\mu_{\mathrm{v}}$ and $\sigma_{\mathrm{v}}$ are the mean and the $\mathrm{SD}$ of $V$, the size of the spine measured in a logarithmic scale (Song et al., 2005; Loewenstein et al., 2011). A positive value of $w_{v}$ implies that larger spines are more stable than smaller ones. The opposite is true if $w_{v}$ is negative.

We used least-squares regression to find the set of parameters $b_{i}$ and $w_{v}$ that best fit the empirically measured survival of the spines. In line with the qualitative analysis of Figure $3 A$, the values of $b_{i}$, depicted in Figure $3 B$, are a monotonously increasing function of the age of the spines, confirming that even when the size of the spine is taken into considerations, the survival probability of the spines increase with their age. The value of $w_{v}=0.49 \pm 0.05$ is positive, indicating that given the age, the survival probability of a spine increases with its size. As depicted in Figure $3 A$ (lines), the spine survival probability model (Eqs. 2, 3) captures the size-age 

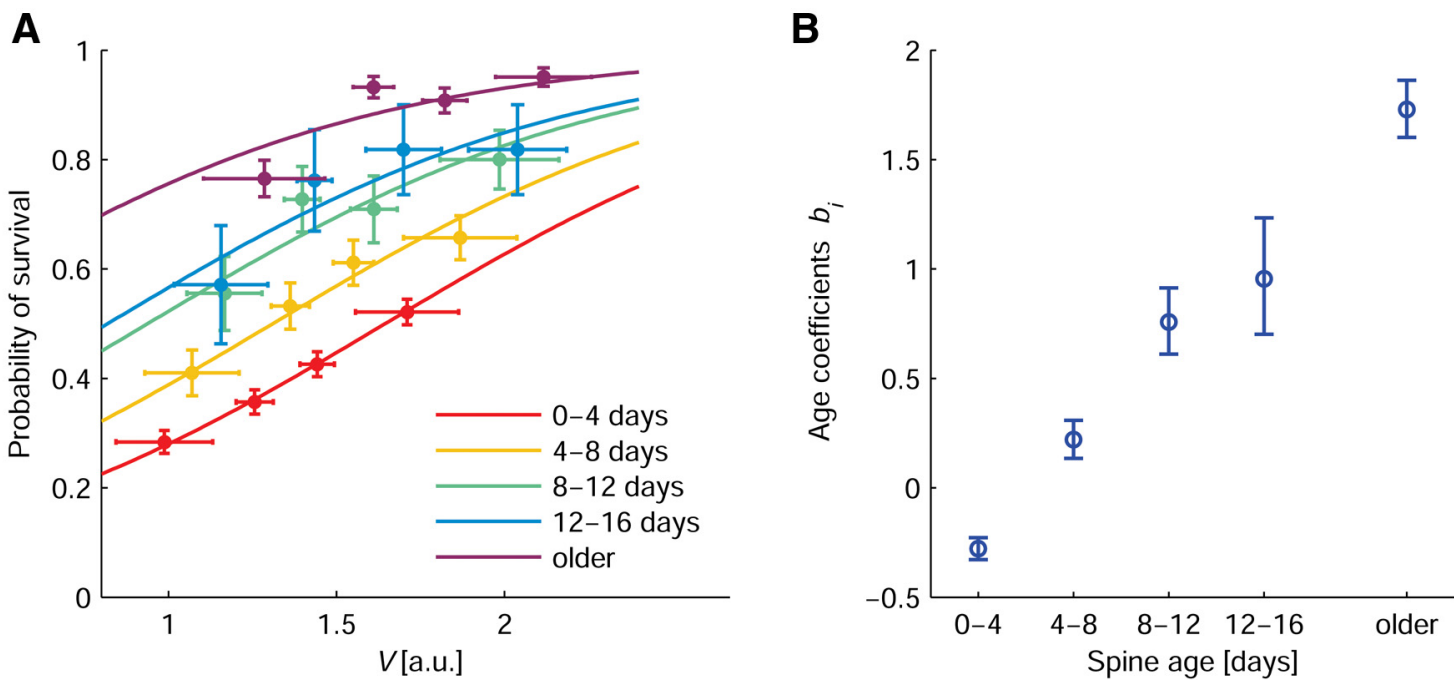

Figure 3. Spine size and age independently determine survival probability. $A$, Circles represent spines of various ages that were grouped into four bins according to their size. For each group, the mean survival probability is plotted against the mean spine size. Error bars on the $x$-axis indicate SD. Error bars on the $y$-axis indicate SEM. Line, Best-fitted model (Eqs. 2,3). $\boldsymbol{B}$, The age coefficients $b_{i} . i=0-4 \mathrm{~d}, 4-8 \mathrm{~d}, 8-12 \mathrm{~d}$, and $12-16 \mathrm{~d}$ represent the spines of known age; $\mathrm{i}=$ "older" indicates the spines present in the first five imaging sessions. Error bars indicate $68 \%$ Cls (equivalent to $1 \mathrm{SD}$ in a normal distribution).

dependence of spine turnover. The horizontal offset of the lines in Figure $3 A$ is determined by the age parameters $b_{i}$, whereas the slope is determined by the size coefficient $w_{v}$.

\section{A comprehensive model based on additional morphological features}

Size is not the only morphological feature of a spine that is available at a single time-point measurement. We considered the relation of additional morphological features with spine turnover. In addition to the size of the spine $V$, we derived a measure of the spine's shape $S$ as reflected in its ellipticity, in which $S=0$ corresponds to a round spine and $S=1$ corresponds to an infinitely thin spine and a measure of its length, the distance $D$ of its center of mass from the dendrite (see Materials and Methods; Fig. 4A). These three variables provide a parametric description of a spine's morphology and they capture the difference between classically defined groups of spines, such as "stubby," "mushroom," or "filopodia-like" (Peters and Kaiserman-Abramof, 1970). We observed a substantial heterogeneity in these three morphological characteristics as depicted in Figure 4, $B$ and $C$.

The predictive power of the size of a spine raises the question of the extent to which the other morphological features (i.e., the shape and the distance) can be used to predict the spines' survival. Addressing this question in a model-free manner is difficult because of the correlations between the features: the pairwise Pearson correlation coefficients between the morphological variables are as follows: $\operatorname{Cor}(V, S)=0.05 ; \operatorname{Cor}(V, D)=0.18$; and $\operatorname{Cor}(S, D)=0.23$. To untangle these correlations, we used the logistic framework (Eq. 2), in which the input $X$ incorporates the age of the spine and the three morphological variables:

$$
X=b_{i}+w_{v} \cdot \tilde{V}+w_{s} \cdot \tilde{S}+w_{d} \cdot \tilde{D}
$$

where $w_{V}, w_{S}$, and $w_{D}$ are coefficients that denote the contributions of the size, shape, and distance, respectively. The tilde are used to denote that these variables are normalized to have zero mean and a unit $\operatorname{SD}\left(\tilde{X}=\frac{A-\mu_{X}}{\sigma_{X}}\right.$ where $\mu_{X}$ and $\sigma_{X}$ are the mean and the SD of $X$, respectively, $X=S, V$, or $D)$. We used the method of least square error to find the set of parameters $b_{i}$, $w_{V}$, $w_{S}$, and $w_{D}$ that best fit the empirically measured survival of the spines to the next imaging session given their age and morphological characteristics. As depicted in Figure 5A, the age coefficients $b_{i}$ increase with the age, demonstrating that, even given the three morphological variables, spines stabilize with their age. The morphological parameters, depicted in Figure $5 B$, indicate that the likelihood of survival of a spine to the next imaging day is dominated by its age and size, yet the shape of a spine also contributes to its survival probability such that the thinner a spine is, the less stable it is. This negative contribution of shape of the spine to its survival probability occurs despite the fact that shape is positively correlated with size (see above). The effect of the distance parameter $D$ on the survival probability of the spine to the next imaging session is small.

\section{Longer-term predictions}

In the previous section, we used the morphological parameters of the spine and its age to predict its survival in the next imaging session ( $4 \mathrm{~d}$ ). However, this framework can be easily extended to predict the lifetime of spines multiple imaging sessions into the future. In that case, the coefficients that denote the contributions of the age, size, shape, and distance become time-dependent, and Equation 4 becomes the following:

$$
X(t)=b_{i}(t)+w_{V}(t) \cdot \tilde{V}+w_{S}(t) \cdot \tilde{S}+w_{D}(t) \cdot \tilde{D}
$$

where the index $t$ denotes the imaging session into the future $(t=$ $1,2, \ldots)$. We denote this model as a "comprehensive model."

To demonstrate this, we considered the 1515 spines imaged in the second imaging session. The survival of the spines present at the second imaging session is depicted in Figure 6A1, where individual spines are sorted according to the age (age $0-4 \mathrm{~d}$ and older, $>4 \mathrm{~d}$ spines). As a result, the index $i$ in Equation 5 takes only two values. The parameters that characterize the comprehensive model are shown in Table 1 (comprehensive model). Focusing on the second imaging day in our dataset as a reference point provides us with partial age information (one interval into 
A
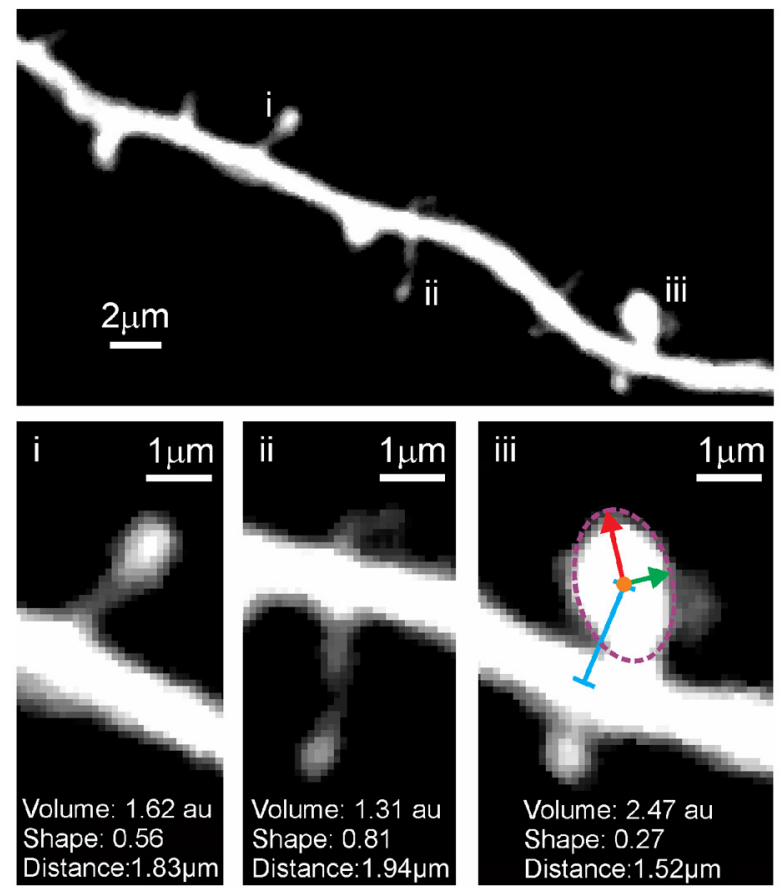

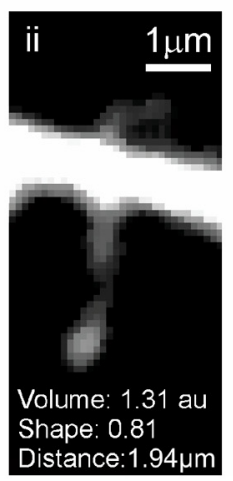

B

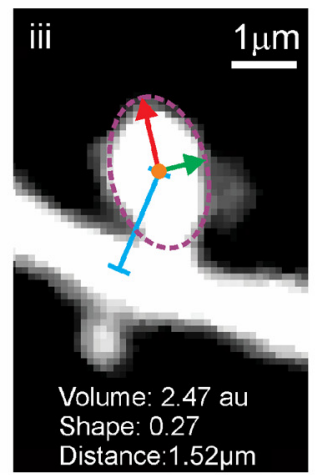

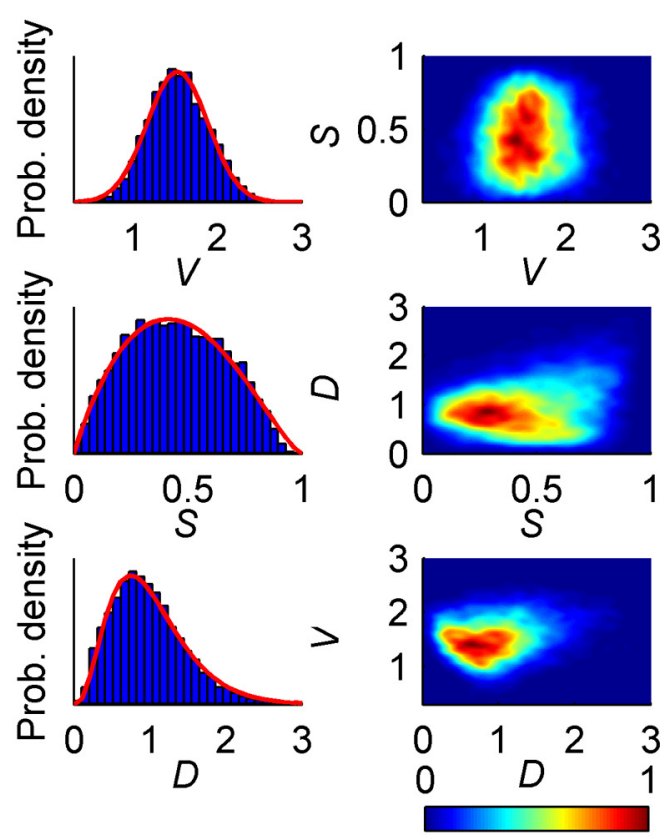

Figure 4. Morphological characteristics of spines. A, Top, "Best projection" image of a dendrite with spines. Bottom, Enlarged views of three example spines with the corresponding morphological parameters describing volume, shape, and distance. For spine iii, orange circle represents the intermediate variables (spine center of mass [COM]. Blue line indicates D, dendrite-COM distance. Purple represents approximating ellipse. Red arrow indicates $\lambda_{1}=$ major axis. Green arrow indicates $\lambda_{2}=$ minor axis. (See Materials and Methods.) $B$, The distributions of the morphological parameters of spines, the size of the spines $V$ (top), the shape variable $S$ (middle), and the distance $D$ (bottom, in $\mu \mathrm{m}$ ). Lines indicate the least-square error fit of a log-normal distribution (top, $\mu_{V}=1.53$ and $\sigma_{V}=0.36$ ) (Loewenstein et al., 2011), beta distribution $f(x ; \alpha, \beta)=\frac{1}{\mathrm{~B}(\alpha, \beta)} x^{\alpha-1}(1-x)^{\beta-1}$ with $\alpha=1.97$ and $\beta=23.7$, and gamma distribution $\left(f(x ; \alpha, \beta)=\frac{\beta^{\alpha}}{\Gamma(\alpha)} x^{\alpha-1} e^{-\beta x}\right.$ with $\alpha=3.80$ and $\left.\beta=26.7\right)$.C, Joint distributions of pairs of parameters, computed by convolving the data points with a Gaussian distribution whose variance is diagonal and is equal to the marginal distribution variances divided by 50 and normalizing maximal probability density to 1 . Top, S versus $D$. Middle, $D$ versus $V$. Bottom, D versus $S$.

A

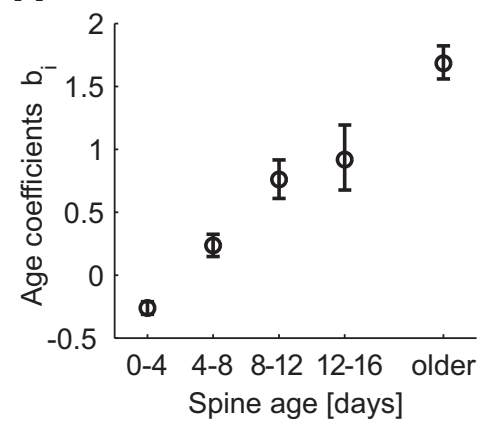

B

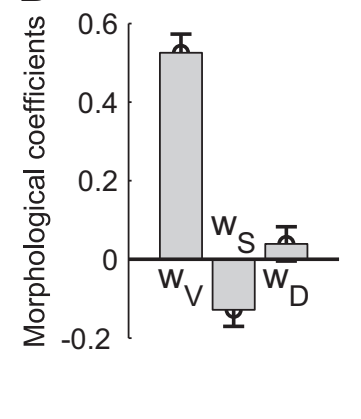

Figure 5. Coefficients of the comprehensive model. $A$, The coefficients for spine age in the comprehensive model. $\boldsymbol{B}$, Coefficients for volume, shape, and distance in the comprehensive model. Values represent the least square error parameters. Error bars indicate $68 \% \mathrm{Cls}$.

the past) as well as reference data for survival predictions up to four imaging intervals into the future.

To illustrate the predictive power of the comprehensive model, we plotted in Figure 6A2, for each spine present in the second imaging session, its survival probability at different intervals into the future. As shown in the figure, the stripes become progressively lighter as the interval increases, reflecting the decrease in survival probability. Also noteworthy is the observation that the lower part of the plot is darker. A standard measure of the predictive power of models is their average log-likelihood. The log-likelihood is nonpositive, and it asymptotically approaches zero the higher the predictive power of the model is. The loglikelihood of the data given the comprehensive model is depicted in Figure $6 B$ (black). A log-likelihood of -1 corresponds to a model that does not do better than the tossing of an unbiased coin. By contrast, the log-likelihood of a model that succeeds in predicting whether a spine will disappear or not with a probability of $80 \%$ is -0.72 (because $0.8 \cdot \log _{2}(0.8)+0.2 \cdot \log _{2}(0.2)=-0.72$ ). It is important to note that we used the leave-one-out cross validation method to compute the likelihood (see Materials and Methods). This method, which is closely related to the Akaike information criterion, was used as it is not biased by overfitting, and it can be used to compare models that are characterized by a different number of parameters (Stone, 1977; Fang, 2011), as is done below. As depicted in Table 1, the magnitudes of the shape and distance coefficients in the comprehensive model are small compared with the magnitude of the size coefficient (qualitatively similar results in Fig. 5B). These results cast doubt on their contribution to the predictive power of the model. To test this, we considered a model that uses only the age and size of the spine (referred to as age-size model). Formally, this model corresponds to assuming that $w_{S}(t)=w_{D}(t)=0$ in Equation 5. The parameters that characterize the age-size model are shown in Table 1 ("age-size"). The predictive power of the age-size model is depicted qualitatively in Figure $6 A 3$ and quantitatively in Figure $6 B$ 
A

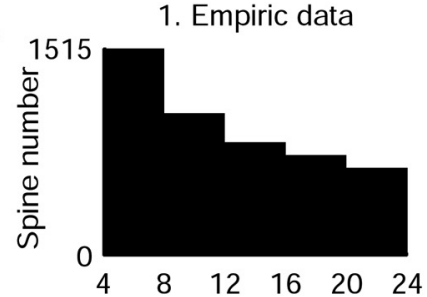

4. ssEM

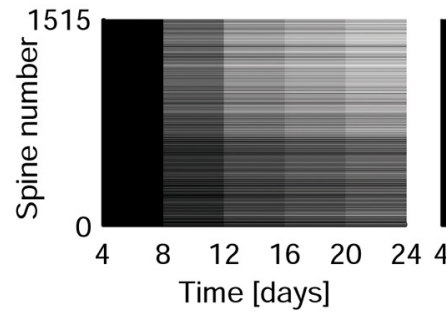

B

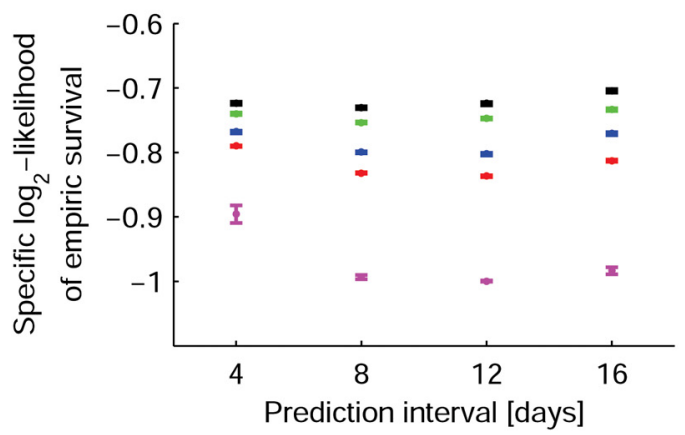

Figure 6. Predicted survival probability of spines. A1, Lifetime plot of all spines present in the second imaging session (spines 1-998 and 1421-1937 in Fig. 1B). As in Fig. 1B, the presence of a spine is indicated by a thin horizontal black, and spines are sorted by the order of their last appearance; spines that last appeared on the same session were randomly shuffled. $\boldsymbol{A} 2$, Predictions of the comprehensive model that takes into account $V, S, D$, and the spines' ages. A3, Predictions of the age-size model that takes into account $V$ and the spins' age. $A 4$, Predictions of the ssEM model that takes into account $V, S$, and $D$. A5, Predictions of the size model that takes into account only $V$. A6, Predictions of a naive model prescribing a constant probability of turnover for all spines. A2-A6, Order of spines is the same as in $\boldsymbol{A} 1$. Grayscale represents the probability of survival. $\boldsymbol{B}$, Specific $\log _{2}$-likelihood of the empirical data $(A 1)$ in view of the five models. Higher likelihood values correspond to a more accurate model. Error bars indicate $68 \% \mathrm{Cls}$. All differences between models are significant $\left(p<10^{-8}\right)$.

(red), demonstrating that the contribution of the shape and distance parameters to the prediction of the fate of the spine, whereas being modest is nevertheless statistically significant $(p<$ $10^{-8}$ for the four intervals; see Materials and Methods).

\section{Emulation of a single time-point ssEM experiment}

In the previous section, we quantified the extent to which the lifetime of a spine can be predicted based on its age and its morphological features. However, in contrast to in vivo imaging studies, the age of the spine is not accessible in single time-point ssEM experiments. Therefore, to test the ability to predict the lifetime of a spine, we considered a model that uses only morphological features of the spine and no explicit information about its age. We considered two models: (1) a model that includes all morphological parameters (formally, this model corresponds to replacing the age-dependent parameters $b_{i}(t)$ in Eq. 5 with an ageindependent constant $b(t))$; we refer to this model as the ssEM model; and (2) a model that considers only the size of the spine, which we refer to as the size model. The parameters that characterize the two models are presented in Table 1 and their predictive power is illustrated qualitatively in Figure $6 A 4, A 5$, respectively, and quantitatively in Figure $6 B$ (blue and brown, respectively). As in Figure $6 A 2, A 3$, the stripes in Figures $6 A 4, A 5$ become progres- sively lighter, moving from the left bottom corner to the right top corner. However, the contrast is slightly lower, reflecting the fact that lack of age information reduces the predictive power of the model. Considering the log-likelihoods of the two models, they are lower than those of the models that incorporate age information $\left(p<10^{-10}\right.$ for each of the two models for each of the four intervals compared with the age-size model, see Materials and Methods). Comparing the ssEM and the size models, the ssEM was significantly better than the size model $\left(p<10^{-10}\right.$ for each of the four intervals; see Materials and Methods), suggesting that the incorporation of the two additional morphological parameters increases the predictive power of the model.

For comparison, we also considered a naive model that makes predictions based on the empirical frequencies in Figure 2B, the survival probabilities that are independent of spine age or morphological features. In this model, the probability of surviving to the $t^{\text {th }}$ imaging session is only a function of $t$ (formally we assume that $w_{V}(t)=w_{S}(t)=w_{D}(t)=0$ and that $b_{i}(t)=b(t)$; see Table 1, "naive"). Because the naive model does not incorporate any spine-specific information, the survival probabilities of all spines are identical, as reflected in the vertical stripes in the lifetime plot (Fig. 6A6).

Comparing the log-likelihood of the data in the five models indicates that the incorporation of the morphological parameters available in an ssEM experiment substantially increases the likelihood of the model and brings it close to a comprehensive model that contains information available only using chronic in vivo two-photon spine imaging. The fact that even just taking into account the size of the spine substantially contributes to the ability to predict the survival of a spine $\left(p<10^{-10}\right.$ for each of the four intervals in comparison with the naive model, see Materials and Methods) indicates that the models can be used to infer structural dynamics from single timepoint experiments.

\section{Discussion}

In our study, we used chronic imaging of dendritic spines in the mouse auditory cortex over a few weeks to study the dynamics of neuronal network connectivity and the extent to which future connectivity can be predicted from information available at a single time-point. We first constructed a comprehensive model that captures the independent contributions of age, size, and other morphological parameters to the survival of a spine. Second, we showed that the morphological features, even in the absence of age information can be used to identify stable and volatile elements. These results demonstrate how data from ssEM experiments can be used to predict future network connectivity. 
Table 1. Parameters and natural likelihoods of different models ${ }^{a}$

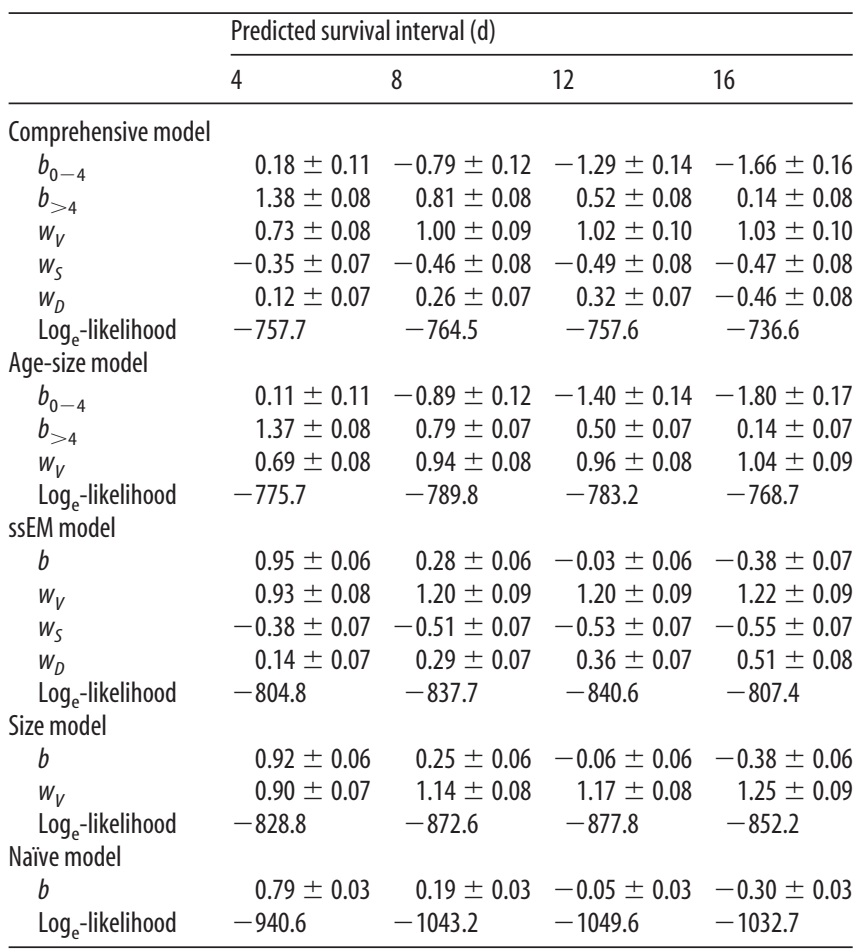

$\bar{a}$ Values represent the least square error parameters. Cls were computed by resampling the spines 10,000 times and computing the $\mathrm{Cl}$ of the distribution of estimated parameters. All parameters were determined by independently fitting each of the five models to the data.

\section{No evidence of categorical classes of dendritic spines}

Spines are often classified into distinct categories according to their stability and according to their morphological features. If dendritic spines can be divided into distinct classes that differ in their turnover dynamics, then, in principle, the identity of a spine within these classes is informative about its lifetime. However, we did not find evidence for distinct spine classes. Regarding their tenacity, in many studies, spines are classified as "stable" if they persist throughout the imaging experiment and "transient" if they appear or disappear during the experiment (Trachtenberg et al., 2002; Holtmaat et al., 2005; Zuo et al., 2005; Majewska et al., 2006; Loewenstein et al., 2011). However, the power law function indicates that spines continuously stabilize with their age at multiple time scales. (Fusi et al., 2005; Yasumatsu et al., 2008; Minerbi et al., 2009). This implies that age-dependent stabilization may be a continuous process; therefore, the often used classification of spines as "stable" or "transient" should be considered an operational definition, rather than reflecting the existence of two distinct populations of spines. Turnover characterized by a power law implies that despite substantial spine elimination, a considerable fraction of spines will last for a long period of time. For example, according to the power law model with $\gamma=1.384$, half of the spines that were present throughout the experiment (lifetime $>20 \mathrm{~d}$ ) were expected to survive for at least another $110 \mathrm{~d}$. Indeed, there is direct experimental evidence for spines in mouse neocortex that are stably maintained for more than a year (Zuo et al., 2005; Yang et al., 2009).

Regarding their morphology, spines are often characterized as "mushroom," "stubby," or "filiopodia-like" (Peters and Kaiserman-Abramof, 1970). We did not find evidence for distinct spine classes: considering the distribution of morphological features as depicted in Figure 4, we did not observe clustering of parameters or combinations of parameters, which could be indicative of distinct spine classes. Therefore, we conclude that the commonly used classification of spines according to their morphology as "“mushroom," "stubby," or "filiopodia-like" represent prototypical examples of a continuum (Holtmaat and Svoboda, 2009). A continuum of morphologies has been reported in a previous study, in which spine morphologies were analyzed, with better resolution, using EM (Arellano et al., 2007a). The lack of morphological classes motivated us to use in our model continuous morphological variables to predict the lifetime of spines.

\section{An emulated ssEM experiment}

It is generally thought that the dynamics of the architecture of neuronal circuits play a crucial role in brain functions, such as learning and long-term storage of memories. However, current techniques for connectomic reconstructions do not allow for measurements at multiple time-points and therefore inherently lack information about network reconfiguration. Here, we tested how far changes in network connectivity can be predicted at a single time-point from particular morphological features of the dendritic spine in the framework of a particular generalized linear model, the logistic regression model. Our study is a first attempt and, as such, sets a lower bound to the predictive power of such an approach. Incorporating nonlinearities in the model may improve its predictive power. For example, additional analysis suggests that shape dependence of turnover is more substantial for thinner spines than it is for rounder spines. Incorporating this and similar results may result in better predictions, as well as being the first step in providing biophysical insights to the dependence of spine survival on the different morphological parameters. It is also conceivable that the inclusion of additional morphological features would improve the predictive power of the model. Any feature of a spine that can be measured in vivo over time and later be correlated with subsequent ssEM measurement can be potentially used to predict the lifetime of a spine in the framework of a connectomic study. Recent connectomic studies have already taken into account not only the presence of a synaptic connection between two neurons but also additional subcellular morphological features, such as the size of the physical contact between two neurons (Helmstaedter et al., 2013). The model for spine longevity based on morphological parameters obtained from two-photon in vivo images can be directly utilized when considering high resolution ssEM data. Furthermore, markers for the molecular composition of a spine could also provide additional valuable information about its stability (Cane et al., 2014).

\section{Volatility and stability in neuronal circuits}

In this study, we identified morphological features that allow the distinction between the more volatile and the more stable elements of a neuronal circuit (Tetzlaff et al., 2012). To what extent does such a distinction help us understand connectivity matrices and provide us with insight into the function of particular circuits?

Conceptually, there are two extreme interpretations with respect to how the changes we observed in the network connectivity affect the resultant network computations. One interpretation is that the changes in spine configuration reflect functional changes in the network. In this case, the changes in the circuit structure may be the physical correlates of the acquisition of new memories or new skills. According to this view, the observed plasticity underlies the extraordinary capacity of the cortex to constantly reconfigure itself in response to new experiences even in the adult 
brain. In this framework, stable aspects of network configurations could either represent older memories and/or essential scaffolds embedded into the circuit to ensure its proper operation.

However, it is also possible that network computations are unaltered despite these changes in synaptic configurations. According to this second interpretation, the synaptic changes are "orthogonal" to the directions of computation. In other words, multiple network configurations are computationally equivalent and the network drifts between these configurations while maintaining the same function (Rokni et al., 2007; Marder, 2011; Shomrat et al., 2011; Ajemian et al., 2013). The fact that a substantial fraction of the changes in network connectivity are predictable by a model that does not take into account external experiences suggests that the changes we observe do not correspond to the ongoing formation of new memories. This is in line with the second interpretation.

The two interpretations are not necessarily mutually exclusive. It is possible that some of the network changes are computationally significant, whereas others are not. One exciting possibility is that the model may help distinguish between the two types of changes: those that are predictable are independent of the external inputs and hence may be computationally insignificant. By contrast, those that are unpredictable may be related to functional changes of the network. The idea is that a fully predictable change in the connectivity cannot reflect the acquisition of novel memories or skills because by definition, if the change is predictable then it does not contain any new information about the environment. An unpredictable change, on the other hand, is potentially (but not necessarily) the outcome of new information stored in the neural system. The extent to which a change in the network architecture is predictable is thus informative about the likelihood that this change is associated with learning.

\section{Notes}

Supplemental material for this article is available at http://bio.huji.ac.il/ yonatanlab/spines/. There, the dataset analyzed in the study, including the morphological parameters of all 3688 spines, is publicly available. This material has not been peer reviewed.

\section{References}

Ajemian R, D'Ausilio A, Moorman H, Bizzi E (2013) A theory for how sensorimotor skills are learned and retained in noisy and nonstationary neural circuits. Proc Natl Acad Sci U S A 110:E5078-E5087. CrossRef Medline

Arellano JI, Benavides-Piccione R, Defelipe J, Yuste R (2007a) Ultrastructure of dendritic spines: correlation between synaptic and spine morphologies. Front Neurosci 1:131-143. CrossRef Medline

Arellano JI, Espinosa A, Fairén A, Yuste R, DeFelipe J (2007b) Non-synaptic dendritic spines in neocortex. Neuroscience 145:464-469. CrossRef Medline

Bargmann CI, Marder E (2013) From the connectome to brain function. Nat Methods 10:483-490. CrossRef Medline

Beaulieu C, Colonnier M (1985) A laminar analysis of the number of roundasymmetrical and flat-symmetrical synapses on spines, dendritic trunks, and cell bodies in area 17 of the cat. J Comp Neurol 231:180-189. CrossRef Medline

Bhatt DH, Zhang S, Gan WB (2009) Dendritic spine dynamics. Annu Rev Physiol 71:261-282. CrossRef Medline

Bock DD, Lee WC, Kerlin AM, Andermann ML, Hood G, Wetzel AW, Yurgenson S, Soucy ER, Kim HS, Reid RC (2011) Network anatomy and in vivo physiology of visual cortical neurons. Nature 471:177-182. CrossRef Medline

Briggman KL, Helmstaedter M, Denk W (2011) Wiring specificity in the direction-selectivity circuit of the retina. Nature 471:183-188. CrossRef Medline

Cane M, Maco B, Knott G, Holtmaat A (2014) The relationship between
PSD-95 clustering and spine stability in vivo. J Neurosci 34:2075-2086. CrossRef Medline

Caroni P, Donato F, Muller D (2012) Structural plasticity upon learning: regulation and functions. Nat Rev Neurosci 13:478-490. CrossRef Medline

Denk W, Briggman KL, Helmstaedter M (2012) Structural neurobiology: missing link to a mechanistic understanding of neural computation. Nat Rev Neurosci 13:351-358. CrossRef Medline

Dickhaus T, Royen T (2015) A survey on multivariate chi-square distributions and their applications in testing multiple hypotheses. Statistics 49: 427-454. CrossRef

Fang Y (2011) Asymptotic equivalence between cross-validations and Akaike information criteria in mixed-effects models. J Data Sci 9:15-21.

Feng G, Mellor RH, Bernstein M, Keller-Peck C, Nguyen QT, Wallace M, Nerbonne JM, Lichtman JW, Sanes JR (2000) Imaging neuronal subsets in transgenic mice expressing multiple spectral variants of GFP. Neuron 28:41-51. CrossRef Medline

Fusi S, Drew PJ, Abbott LF (2005) Cascade models of synaptically stored memories. Neuron 45:599-611. CrossRef Medline

Grutzendler J, Kasthuri N, Gan WB (2002) Long-term dendritic spine stability in the adult cortex. Nature 420:812-816. CrossRef Medline

Helmstaedter M, Briggman KL, Turaga SC, Jain V, Seung HS, Denk W (2013) Connectomic reconstruction of the inner plexiform layer in the mouse retina. Nature 500:168-174. CrossRef Medline

Holtmaat A, Svoboda K (2009) Experience-dependent structural synaptic plasticity in the mammalian brain. Nat Rev Neurosci 10:647-658. CrossRef Medline

Holtmaat AJ, Trachtenberg JT, Wilbrecht L, Shepherd GM, Zhang X, Knott GW, Svoboda K (2005) Transient and persistent dendritic spines in the neocortex in vivo. Neuron 45:279-291. CrossRef Medline

Holtmaat A, Wilbrecht L, Knott GW, Welker E, Svoboda K (2006) Experience-dependent and cell-type-specific spine growth in the neocortex. Nature 441:979-983. CrossRef Medline

Holtmaat A, Bonhoeffer T, Chow DK, Chuckowree J, De Paola V, Hofer SB, Hübener M, Keck T, Knott G, Lee WC, Mostany R, Mrsic-Flogel TD, Nedivi E, Portera-Cailliau C, Svoboda K, Trachtenberg JT, Wilbrecht L (2009) Long-term, high-resolution imaging in the mouse neocortex through a chronic cranial window. Nat Protoc 4:1128-1144. CrossRef Medline

Hübener M, Bonhoeffer T (2010) Searching for engrams. Neuron 67:363371. CrossRef Medline

Kasai H, Fukuda M, Watanabe S, Hayashi-Takagi A, Noguchi J (2010) Structural dynamics of dendritic spines in memory and cognition. Trends Neurosci 33:121-129. CrossRef Medline

Keck T, Mrsic-Flogel TD, Vaz Afonso M, Eysel UT, Bonhoeffer T, Hübener M (2008) Massive restructuring of neuronal circuits during functional reorganization of adult visual cortex. Nat Neurosci 11:1162-1167. CrossRef Medline

Knott GW, Holtmaat A, Wilbrecht L, Welker E, Svoboda K (2006) Spine growth precedes synapse formation in the adult neocortex in vivo. Nat Neurosci 9:1117-1124. CrossRef Medline

Lichtman JW, Denk W (2011) The big and the small: challenges of imaging the brain's circuits. Science 334:618-623. CrossRef Medline

Loewenstein Y, Kuras A, Rumpel S (2011) Multiplicative dynamics underlie the emergence of the log-normal distribution of spine sizes in the neocortex in vivo. J Neurosci 31:9481-9488. CrossRef Medline

Majewska AK, Newton JR, Sur M (2006) Remodeling of synaptic structure in sensory cortical areas in vivo. J Neurosci 26:3021-3029. CrossRef Medline

Marder E (2011) Variability, compensation, and modulation in neurons and circuits. Proc Natl Acad Sci U S A 108 [Suppl 3]:15542-15548.

Minerbi A, Kahana R, Goldfeld L, Kaufman M, Marom S, Ziv NE (2009) Long-term relationships between synaptic tenacity, synaptic remodeling, and network activity. PLoS Biol 7:e1000136. CrossRef Medline

Moczulska KE, Tinter-Thiede J, Peter M, Ushakova L, Wernle T, Bathellier B, Rumpel S (2013) Dynamics of dendritic spines in the mouse auditory cortex during memory formation and memory recall. Proc Natl Acad Sci U S A 110:18315-18320. CrossRef Medline

Peters A, Kaiserman-Abramof IR (1970) The small pyramidal neuron of the rat cerebral cortex: the perikaryon, dendrites and spines. Am J Anat 127: 321-355. CrossRef Medline

Rokni U, Richardson AG, Bizzi E, Seung HS (2007) Motor learning with 
unstable neural representations. Neuron 54:653-666. CrossRef Medline

Seung HS (2009) Reading the book of memory: sparse sampling versus dense mapping of connectomes. Neuron 62:17-29. CrossRef Medline

Shomrat T, Graindorge N, Bellanger C, Fiorito G, Loewenstein Y, Hochner B (2011) Alternative sites of synaptic plasticity in two homologous "fanout fan-in" learning and memory networks. Curr Biol 21:1773-1782. CrossRef Medline

Song S, Sjöström PJ, Reigl M, Nelson S, Chklovskii DB (2005) Highly nonrandom features of synaptic connectivity in local cortical circuits. PLoS Biol 3:e68. CrossRef Medline

Stone M (1977) An asymptotic equivalence of choice of model by crossvalidation and Akaike's criterion. J R Stat Soc B 39:44-47.

Takemura SY, Bharioke A, Lu Z, Nern A, Vitaladevuni S, Rivlin PK, Katz WT, Olbris DJ, Plaza SM, Winston P, Zhao T, Horne JA, Fetter RD, Takemura S, Blazek K, Chang LA, Ogundeyi O, Saunders MA, Shapiro V, Sigmund C, et al. (2013) A visual motion detection circuit suggested by Drosophila connectomics. Nature 500:175-181. CrossRef Medline

Tetzlaff C, Kolodziejski C, Markelic I, Wörgötter F (2012) Time scales of memory, learning, and plasticity. Biol Cybern 106:715-726. CrossRef Medline

Trachtenberg JT, Chen BE, Knott GW, Feng G, Sanes JR, Welker E, Svoboda
K (2002) Long-term in vivo imaging of experience-dependent synaptic plasticity in adult cortex. Nature 420:788-794. CrossRef Medline

White JG, Southgate E, Thomson JN, Brenner S (1986) The structure of the nervous system of the nematode Caenorhabditis elegans. Philos Trans R Soc Lond B Biol Sci 314:1-340. CrossRef Medline

Wilks SS (1938) The large-sample distribution of the likelihood ratio for testing composite hypotheses. Ann Math Statist 9:60-62. CrossRef

Xu T, Yu X, Perlik AJ, Tobin WF, Zweig JA, Tennant K, Jones T, Zuo Y (2009) Rapid formation and selective stabilization of synapses for enduring motor memories. Nature 462:915-919. CrossRef Medline

Yang G, Pan F, Gan WB (2009) Stably maintained dendritic spines are associated with lifelong memories. Nature 462:920-924. CrossRef Medline

Yasumatsu N, Matsuzaki M, Miyazaki T, Noguchi J, Kasai H (2008) Principles of long-term dynamics of dendritic spines. J Neurosci 28:1359213608. CrossRef Medline

Zheng P, Dimitrakakis C, Triesch J (2013) Network self-organization explains the statistics and dynamics of synaptic connection strengths in cortex. PLoS Comput Biol 9:e1002848. CrossRef Medline

Zuo Y, Lin A, Chang P, Gan WB (2005) Development of long-term dendritic spine stability in diverse regions of cerebral cortex. Neuron 46:181189. CrossRef Medline 